\title{
IMPORTANCIA RELATIVA DE LOS DISTINTOS MODOS DE TRANSPORTE, CON ESPECIAL REFERENCIA AL TRAFICO POR CARRETERA Y TRANSPORTE URBANO
}

656.05: 711.7

por

Ignacio Ballester Ros

SUMARIO: I. INTRODUCCION.-II. IMPORTANCIA RELATIVA DEL TRANSPORTE Y SUS DISTINTOS MODOS.-III. LA RED DE CARRETERAS Y EL TRAFICO QUE SOPORTA.IV. EL TRANSPORTE URBANO.

\section{INTRODUCCION}

Los Ministerios de Obras Públicas y Urbanismo y de Transportes y Comunicaciones han publicado recientemente tres monografías con un contenido de extraordinario interés, que permite conocer la estructura y evolución de las obras públicas, del urbanismo y del transporte, en los últimos años.

La primera de ellas, bajo el título de Las obras públicas y el urbanismo en 1978. MOPU. Información estadistica, comprende la visión actualizada de las inversiones, realizaciones y explotaciones llevadas a cabo en dicho año, así como las obras en curso en carreteras, puertos y costas, obras hidráulicas, arquitectura y vivienda, urbanismo, ordenación y acción territorial y medio ambiente, con una referencia especial a las acciones específicas de los Institutos Nacionales de la Vivienda y de Urbanismo. 
La segunda es la Memoria del Ejercicio de 1978 del Instituto de Estudios de Transportes y Comunicaciones, actualmente adscrito al Ministerio de Transportes y Comunicaciones, y continuador de la labor del antiguo Consejo Superior de Transportes, cuyas competencias y funciones ha asumido y ampliado, monografía en la que se presentan las características estructurales del sector del transporte y su actividad en el año de referencia.

El propio Instituto de Estudios de Transportes y Comunicaciones ha editado otra monografía sobre Estadisticas de transportes. Series cronológicas (1950-1977), que nos ofrece una perspectiva suficientemente amplia para el análisis de la evolución del sector en los últimos decenios.

Se trata de tres monografías, en cierto modo complementarias, pues si las dos últimas se dedican especialmente al estudio del transporte, la primera, con su amplio campo de examen de las obras públicas, cubre el análisis del soporte del transporte terrestre, marítimo, ferroviario y aéreo, es decir, de las carreteras, ferrocarriles, puertos y aeropuertos y otras obras públicas, entre ellas, los oleoductos y gaseoductos.

La información estadística, elaborada por distintos organismos dependientes de ambos Ministerios, en especial, por el Instituto de Estudios de Transportes y Comunicaciones y, también, por el Instituto Nacional de Estadística, es muy amplia, al propio tiempo que profundiza en el análisis de cada uno de los modos de transporte.

Entre la extensa gama de materias a que se extienden estas monografías, elegimos, como tema base de este comentario, el transporte en sus líneas generales, con especial referencia a su importancia relativa en la economía nacional, al transporte por carretera y al transporte urbano.

La proyección del transporte es, hoy en día, triple. De una parte, hay que resaltar su perspectiva internacional, de valor decisivo en el momento en que mantenemos negociaciones para nuestra entrada en la Comunidad Económica Europea, cuya legislación en este sector habremos de tener muy en cuenta. En segundo lugar, su perspectiva nacional, como uno de los principales soportes de la actividad económica. $Y$, en tercer término, la perspectiva del transporte urbano, dentro de las grandes urbes y su prolongación a las comarcas inmediatas, que constituyen sus zonas de influencia y facilita el tráfico diario, dando origen a las migraciones alternantes. 


\section{IMPORTANCIA RELATIVA DEL TRANSPORTE Y SUS DISTINTOS MODOS}

Varios son los indicadores que permiten conocer la importancia del transporte en la economía nacional y el valor relativo de los distintos modos de transporte al servicio de la actividad humana.

El sector del transporte participó, en 1975, en el 5,7 por $100 \mathrm{del}$ Producto Interior Bruto. La serie anual, en el período 1954-1975, es bastante irregular. En 1954 esta participación fue del 5,4; en el período 1956-58 se eleva al 5,6; disminuye en el siguiente bienio y aún más en 1961-1964; el quinquenio 1965-1969 es el de mayor participación, llegando al 5,9; pero nuevamente se debilita en el siguiente quinquenio.

La participación de los distintos modos de transporte en la composición del PIB del sector, es como sigue:

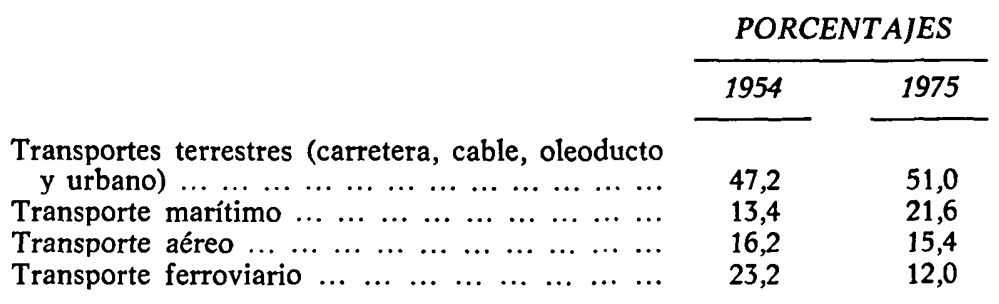

Como se observa, en el periodo 1954-1975 se ha registrado un decrecimiento relativo muy acusado, reduciéndose casi a la mitad, del transporte ferroviario; un ligero descenso del transporte aéreo; un incremento de los transportes terrestres $\mathrm{y}$, tal vez, más acentuado, del transporte marítimo.

La Formación Bruta de Capital Fijo en materia móvil, en 1970, representó en el sector del transporte el 11,4 por 100 de la FBCF total, participación que desciende ligeramente en 1975, al 11,2.

Los Gastos del Estado y de los Organismos Autónomos en este sector durante el período 1971-1977, expresados en porcentajes del gasto total, han sido:

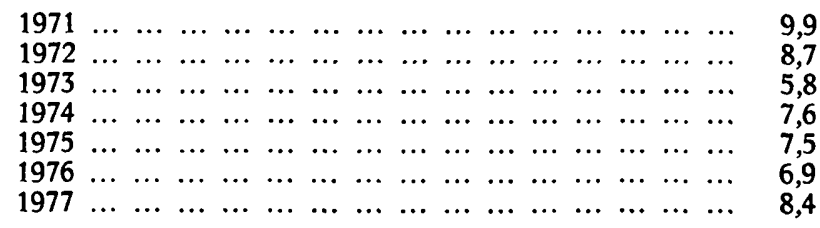


Como se advierte, la tendencia en el citado período ha sido decreciente, con unos valores mínimos en 1973 y 1976 y otros máximos en 1971-72 y 1977.

En términos absolutos, los gastos crecieron en un 67 por 100 , incrementos que afectaron más a los transportes marítimos (95 por 100), al ferroviario (71) y por carretera (64).

Las inversiones realizadas en infraestructura, en 1971 y 1977, han sido, por modos de transporte:

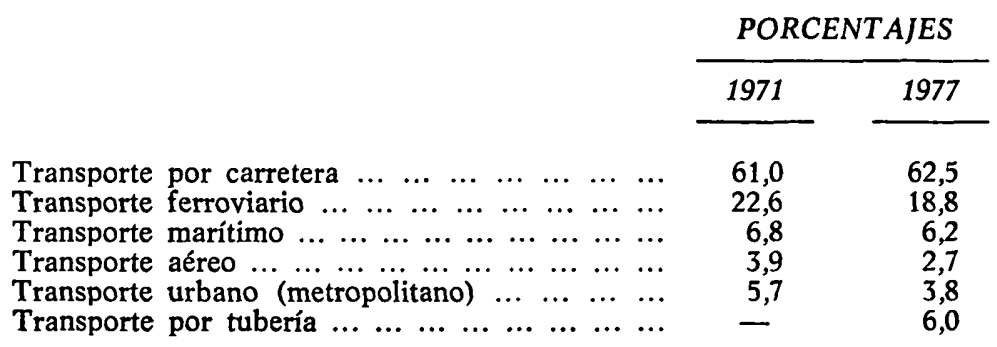

Se observa un aumento de las inversiones aplicadas al transporte por carretera y una disminución generalizada de las inversiones en los demás modos de transporte. También se registra la aparición de un nuevo modo de transporte, el por tubería, que comprende a los oleoductos y a los gaseoductos, cuya importancia se pone de manifiesto por lo que a petróleos brutos y refinados y a gases se refiere.

La población ocupada en el sector fue, en 1973, de 654.531 personas y en 1977 se elevó a 708.540, con un incremento del 8,2 por 100 .

La distribución de esta población ocupada, o lo que es lo mismo, del personal empleado en el transporte, por modos de transporte, fue, en 1977, la siguiente:

Porcentajes

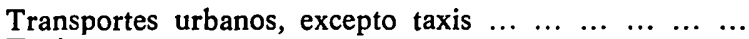

$\begin{array}{lllllllllllllllll}\text { Taxis } & \ldots & \ldots & \ldots & \ldots & \ldots & \ldots & \ldots & \ldots & \ldots & \ldots & \ldots & \ldots & \ldots & \ldots & \ldots\end{array}$

Transporte regular de viajeros por carretera $\ldots \ldots \ldots \ldots$

Transporte discrecional de viajeros por carretera .......

Transporte público de mercancías por carretera ... ...

Transporte ferroviario. Renfe $\ldots \ldots \ldots \ldots \ldots \ldots$

Transporte ferroviario. Feve y otros ferrocarriles de vía estrecha

$\left.\begin{array}{r}5,1 \\ 10,1\end{array}\right\} 15,2$

$\left.\begin{array}{r}5,3 \\ 2,3 \\ 61,8\end{array}\right\} 69,4$

10,2

$1,0\} 11,2$

$\begin{array}{llllllllllllll}\text { Transporte marítimo } & \ldots & \ldots & \ldots & \ldots & \ldots & \ldots & \ldots & \ldots & \ldots & \ldots & \ldots & & 3,0\end{array}$

$\begin{array}{llllllllllllll}\text { Transporte aéreo } & \ldots & \ldots & \ldots & \ldots & \ldots & \ldots & \ldots & \ldots & \ldots & \ldots & \ldots & \ldots & \end{array}$ 
Es de destacar que el personal empleado en los transportes por carretera supera las dos terceras partes del total. Le siguen en importancia, aunque a lo lejos, los transportes urbanos, con una sexta parte, seguidos del transporte ferroviario, con una décima parte. Los transportes marítimo y aéreo, con valores similares, completan la población activa en el sector.

En el período 1973-1977, los incrementos de personal ocupado, por modos de transporte, han sido:

\begin{tabular}{|c|c|}
\hline & Porcentajes \\
\hline $\begin{array}{l}\text { Transportes urbanos, excepto } \text { taxis } \ldots \\
\text { Taxis }{ }^{2}\end{array}$ & $\begin{array}{r}7,1 \\
179\end{array}$ \\
\hline Transporte regular de viajeros por carretera $\ldots \ldots \ldots \ldots$ & 1,6 \\
\hline Transporte discrecional de viajeros por carretera ... . ... & 13,3 \\
\hline Transporte público de mercancías por carretera ... ... & 8,2 \\
\hline Transporte ferroviario $\operatorname{Renfe}\left(\begin{array}{llllllll} & \ldots & \ldots & \ldots & \ldots & \ldots & \ldots & \ldots\end{array}\right.$ & $-0,8$ \\
\hline Transporte ferroviario. Feve y otros ferrocarriles de vía & 198 \\
\hline Transporte maritimo $\ldots \ldots \ldots$ & $\begin{array}{r}-13,0 \\
22,8\end{array}$ \\
\hline Transporte aéreo ...... & 19,2 \\
\hline
\end{tabular}

Los aumentos de personal ocupado fueron muy significativos en el período de referencia. A los transportes marítimo y aéreo corresponden los mayores incrementos, una quinta parte en cada uno, seguidos de los conductores de taxis y el personal de transporte discrecional de viajeros por carretera. Es de señalar la disminución de personal de la Renfe y, muy especialmente, de los ferrocarriles de vía estrecha, que han perdido la quinta parte de su personal en el período señalado.

El tráfico interior de viajeros y de mercancías, en 1975 y en 1978 ha sido, expresado en porcentajes:

\begin{tabular}{|c|c|c|c|c|}
\hline & \multicolumn{2}{|c|}{ VIAJEROS } & \multicolumn{2}{|c|}{ MERCANCIAS } \\
\hline & 1975 & 1978 & 1975 & 1978 \\
\hline $\begin{array}{llllllllll}\text { Por carretera } & \ldots & \ldots & \ldots & \ldots & \ldots & \ldots & \ldots & \ldots \\
\text { Por ferrocarril } & \ldots & \ldots & \ldots & \ldots & \ldots & \ldots & \ldots & \ldots & \ldots \\
\text { Marítimo } & \ldots & \ldots & \ldots & \ldots & \ldots & \ldots & \ldots & \ldots & \ldots \\
\text { Méreo } & \ldots & \ldots & \ldots & \ldots & \ldots & \ldots & \ldots & \ldots & \ldots \\
\text { Por tubería } & \ldots & \ldots & \ldots & \ldots & \ldots & \ldots & \ldots & \ldots \\
\text { Por tuberian } & \ldots & \ldots & \ldots & \ldots & \ldots & \ldots & \ldots\end{array}$ & $\begin{array}{l}85,7 \\
11,7 \\
2,6\end{array}$ & $\begin{array}{l}86,2 \\
10,4 \\
3,4\end{array}$ & $\begin{array}{r}64,9 \\
9,4 \\
23,8 \\
0,1 \\
1,8\end{array}$ & $\begin{array}{r}67,7 \\
8,2 \\
22,1 \\
0,1 \\
2,0\end{array}$ \\
\hline
\end{tabular}

El transporte por carretera es el predominante, ya que representa las cinco sextas partes del tráfico total de viajeros y alrededor de los dos tercios del de mercancías. En segundo lugar figura el tráfico marítimo, en cuanto a mercancías se refiere, ya que absorbe más de la quinta parte de dicho tráfico. En tercer término figura 
el tráfico ferroviario, con la décima parte del movimiento de viajeros y algo menos de mercancías y, finalmente, el aéreo, de menor importancia relativa, tanto con respecto al tráfico de viajeros como al de mercancías.

En el período 1975-1978 se ha registrado un incremento del tráfico por carretera y aéreo de viajeros, así como del transporte de mercancías por carretera; e igualmente, del transporte de crudos y refinados por tubería. Los demás tráficos han experimentado ligeros descensos.

En cuanto al tráfico internacional en 1978, ha sido:

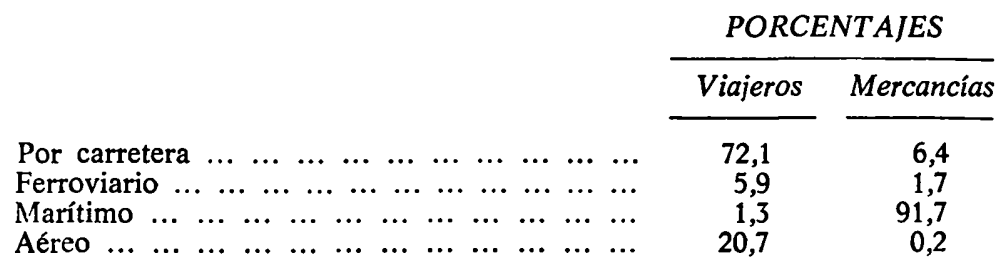

Como se deduce del examen de las cifras anteriores, el tráfico internacional de viajeros se realiza básicamente por carretera y por vía aérea, mientras que el tráfico de mercancías es muy predominantemente marítimo.

\section{LA RED DE CARRETERAS Y EL TRAFICO QUE SOPORTA}

En 1978, la red de carreteras tenía una longitud de $148.331,9$ kilómetros, que se descomponen de la siguiente forma:

\begin{tabular}{|c|c|}
\hline & $\begin{array}{l}\text { Longitud } \\
\text { en kms. }\end{array}$ \\
\hline 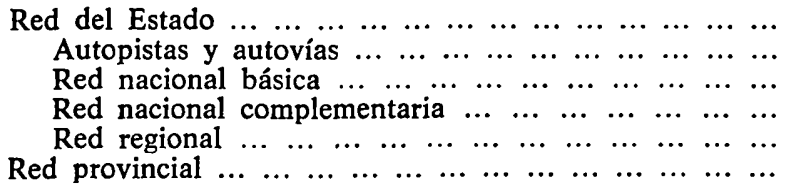 & $\begin{array}{r}81.597,3 \\
1.724,7 \\
16.185,1 \\
18.942,3 \\
44.745,2 \\
66.734,6\end{array}$ \\
\hline
\end{tabular}

Como se observa, la red del Estado representa el 55 por 100 de la longitud total de las carreteras españolas. La red nacional abarca la cuarta parte de la red estatal y la red regional el 30 por 100. La longitud de las autopistas y autovías supone, tan sólo, de momento, el 1,2 por 100 de la red estatal. De otra parte, la red nacional básica representa el 11 por 100 y la complementaria el 13 por 100. La red 
provincial, constituida por las carreteras provinciales y los caminos vecinales, abarca el 45 por 100 de las carreteras españolas.

A continuación se detalla, por Provincias, la distribución de las expresadas redes, con una tabla especial para las autopistas y autovías.

\section{TABLA I.-AUTOPISTAS Y AUTOVIAS ABIERTAS AL TRAFICO EN 1978 (kms.)}

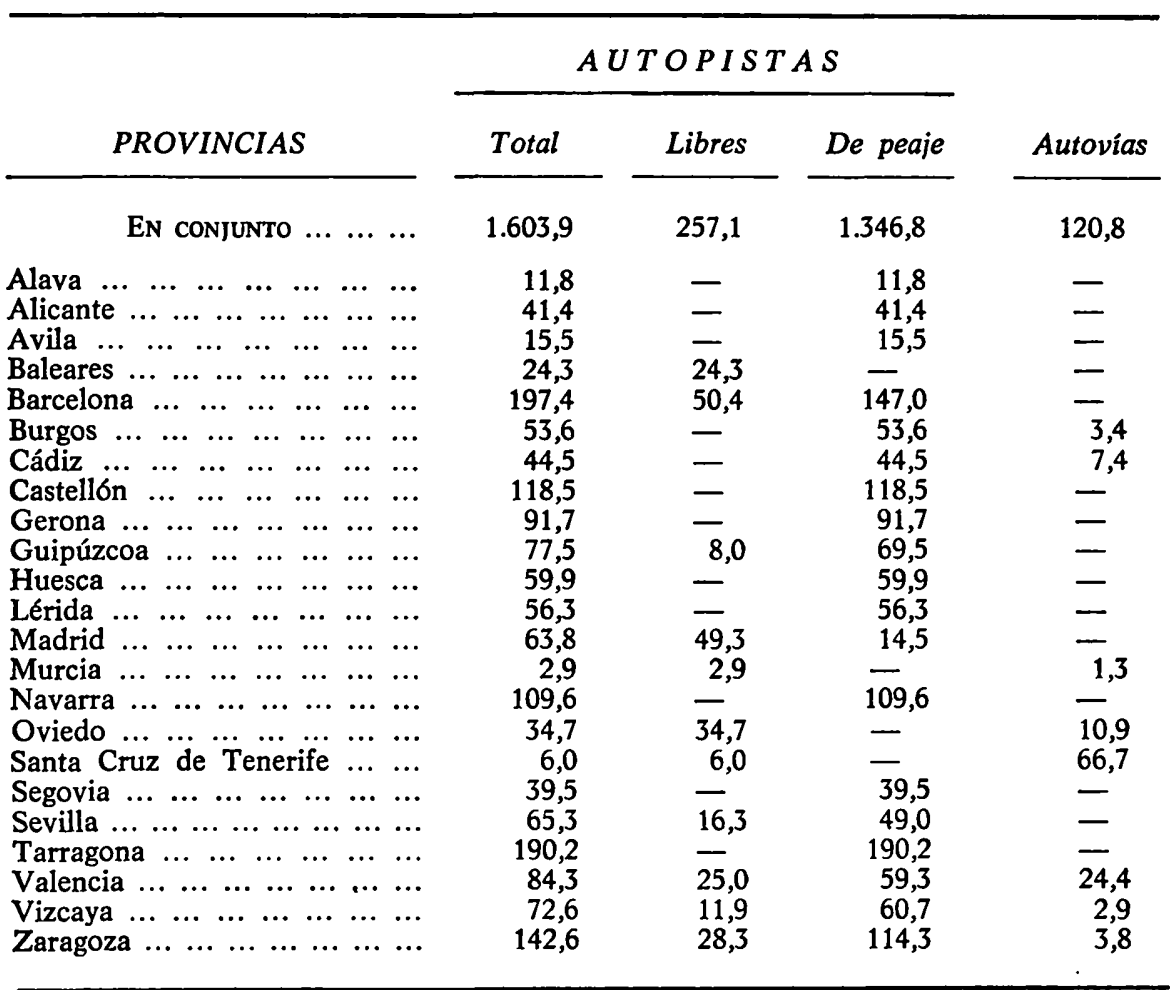

La red de autopistas ha aumentado rápidamente en los últimos años. Predominan las de peaje, que representan el 84 por 100 de la longitud total. Las autopistas en servicio cruzan 23 Provincias. La más importante es la que va desde la frontera, en Gerona, hasta Murcia, a lo largo del litoral mediterráneo. La segunda, por su longitud, comunica a Cataluña con Navarra, las tres Provincias vascas y Burgos, a través del valle del Ebro. Madrid es el centro de una red, corta en longitud, pero densa en tráfico, que se adentra en las Provincias de Segovia y Avila. En las Provincias de Cádiz 
y Sevilla, Oviedo, Baleares y Tenerife existen algunos tramos de autopistas. Las autovías en funcionamiento se limitan hoy a ocho Provincias, destacando por su mayor longitud las de Tenerife y Valencia.

TABLA II.-RED DE CARRETERAS (EXCEPTO AUTOPISTAS Y AUTOVIAS) EN 1978 (kms.)

\begin{tabular}{|c|c|c|c|c|c|}
\hline \multirow[b]{2}{*}{ PROVINCIAS } & \multicolumn{2}{|c|}{ RED NACIONAL } & \multirow[b]{2}{*}{$\begin{array}{l}\text { Red } \\
\text { regional }\end{array}$} & \multirow[b]{2}{*}{$\begin{array}{c}\text { Red } \\
\text { provincial }\end{array}$} & \multirow[b]{2}{*}{ Total } \\
\hline & Básica & $\begin{array}{l}\text { Comple- } \\
\text { mentaria }\end{array}$ & & & \\
\hline $\begin{array}{lllll}\text { Alava } & \ldots & \ldots & \ldots & \ldots\end{array}$ & - & - & - & $1.226,4$ & $1.226,4$ \\
\hline Albacete... ... ... ... & 519,7 & 323,6 & $1.039,7$ & $1.460,6$ & $3.343,6$ \\
\hline Alicante .......... & 301,9 & 299,4 & 889,7 & 912,6 & $2.403,6$ \\
\hline Almería ... ........ & 278,3 & 282,0 & 446,2 & $1.170,9$ & $2.177,4$ \\
\hline Avila... ......... & 173,3 & 449,5 & 758,2 & 896,0 & $2.277,0$ \\
\hline Badajoz ........... & 688,8 & 413,2 & $1.294,8$ & $2.134,0$ & $4.530,8$ \\
\hline Baleares ... ........ & 161,2 & 442,9 & 737,5 & 755,5 & $2.097,1$ \\
\hline Barcelona $\ldots \ldots \ldots$ & 402,7 & 332,4 & 689,4 & $1.908,5$ & $3.333,0$ \\
\hline Burgos $\ldots \ldots \ldots$ & 338,6 & 583,5 & $1.627,3$ & $2.090,8$ & $4.640,2$ \\
\hline Cáceres $\ldots \ldots \ldots \ldots$ & 409,3 & 513,5 & $1.007,7$ & $1.838,0$ & $3.768,5$ \\
\hline Cádiz $\ldots \ldots \ldots \ldots$ & 328,2 & 338,4 & 637,5 & 658,4 & $1.962,5$ \\
\hline Castellón... .......... & 251,6 & 250,7 & 787,7 & 782,9 & $2.072,9$ \\
\hline Ciudad Real ... ... & 414,1 & 597,8 & $1.317,1$ & $1.358,2$ & $3.867,2$ \\
\hline Córdoba... $\ldots \ldots \ldots$ & 349,1 & 508,3 & $1.120,0$ & $2.303,1$ & $4.280,5$ \\
\hline Coruña, La... ... ... & 192,4 & 647,0 & $1.045,8$ & $1.863,0$ & $3.748,2$ \\
\hline Cuenca $\ldots \ldots \ldots$...... & 457,2 & 520,6 & $1.325,3$ & $1.252,5$ & $3.555,6$ \\
\hline Gerona $\ldots \ldots \ldots$ & 278,6 & 321,5 & 883,5 & 724,9 & $2.208,5$ \\
\hline $\begin{array}{lllll} & \\
\text { Granada... } & \ldots & \ldots & \ldots & \ldots\end{array}$ & 535,6 & 400,6 & 712,4 & $1.200,5$ & $2.849,1$ \\
\hline Guadalajara... ... ... & 192,9 & 438,1 & $1.356,3$ & $1.146,0$ & $3.133,3$ \\
\hline Guipúzcoa ... ... ... & 173,9 & 137,2 & 125,1 & 739,0 & $1.175,2$ \\
\hline 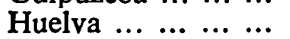 & 293,4 & 333,7 & 444,8 & 890,5 & $1.962,4$ \\
\hline Huesca $\ldots \ldots \ldots \ldots$ & 472,4 & 454,2 & $1.701,4$ & 489,3 & $3.117,3$ \\
\hline Jaén .............. & 324,0 & 475,2 & 885,8 & $1.732,0$ & $3.417,0$ \\
\hline León $\ldots \ldots \ldots \ldots \ldots$ & 435,0 & 586,0 & $1.251,9$ & $1.830,4$ & $4.103,3$ \\
\hline $\begin{array}{lllll}\text { Lerida } & \ldots & \ldots & \ldots & \ldots\end{array}$ & 441,3 & 424,4 & 804,9 & 845,9 & $2.516,5$ \\
\hline Logroño $\ldots \ldots \ldots \ldots$ & 193,2 & 199,2 & 644,2 & 526,8 & $1.563,4$ \\
\hline 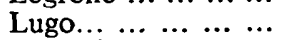 & 369,9 & 381,9 & $1.014,6$ & $2.649,5$ & $4.415,9$ \\
\hline Madrid $\ldots \ldots \ldots \ldots$ & 768,3 & 502,0 & 834,6 & $1.322,1$ & $3.427,0$ \\
\hline Málaga ... ... ... ... & 346,7 & 372,4 & 670,0 & 736,9 & $2.126,0$ \\
\hline $\begin{array}{lllll}\text { Murcia } & \ldots & \ldots & \ldots & \ldots\end{array}$ & 237,4 & 543,5 & 907,2 & $1.576,6$ & $3.264,7$ \\
\hline Navarra ... ... ... ... & - & - & - & $3.417,9$ & $3.417,9$ \\
\hline Orense $\ldots \ldots \ldots \ldots$ & 259,3 & 267,1 & 783,7 & $1.613,7$ & $2.923,8$ \\
\hline $\begin{array}{lllll}\text { Oviedo } & \ldots & \ldots & \ldots & \ldots\end{array}$ & 391,3 & 630,0 & $1.661,6$ & $2.226,6$ & $4.909,5$ \\
\hline Palencia ... ......... & 242,6 & 343,6 & $1.230,6$ & 949,2 & $2.766,0$ \\
\hline Palmas, Las ... ... & 130,2 & 220,7 & 432,6 & $1.248,3$ & $2.031,8$ \\
\hline Pontevedra... $\ldots \ldots$ & 208,7 & 156,7 & $1.135,7$ & $1.491,1$ & $2.992,2$ \\
\hline Salamanca $\ldots \ldots$. & 303,7 & 232,3 & $1.034,0$ & $1.979,0$ & $3.549,0$ \\
\hline S. C. de Tenerife... & 191,6 & 380,2 & 362,9 & 952,2 & $1.886,9$ \\
\hline Santander $\ldots \ldots \ldots$ & 252,9 & 283,4 & $1.010,7$ & 882,4 & $2.429,4$ \\
\hline Segovia $\ldots \ldots \ldots \ldots$ & 204,6 & 441,3 & 436,3 & $1.221,0$ & $2.303,2$ \\
\hline $\begin{array}{lllll}\text { Sevilla } & \ldots & \ldots & \ldots & \ldots\end{array}$ & 432,7 & 739,2 & 888,9 & $1.682,3$ & $3.743,1$ \\
\hline $\begin{array}{lllll}\text { Soria } & \ldots & \ldots & \ldots & \ldots\end{array}$ & 307,5 & 195,1 & 830,1 & $1.188,0$ & $2.520,7$ \\
\hline Tarragona $\ldots \ldots \ldots$ & 188,4 & 327,2 & 859,5 & 984,1 & $2.359,2$ \\
\hline
\end{tabular}




\begin{tabular}{|c|c|c|c|c|c|}
\hline \multirow[b]{2}{*}{ PROVINCIAS } & \multicolumn{2}{|c|}{ RED NACIONAL } & \multirow[b]{2}{*}{$\begin{array}{l}\text { Red } \\
\text { regional }\end{array}$} & \multirow[b]{2}{*}{$\begin{array}{c}\text { Red } \\
\text { provincial }\end{array}$} & \multirow[b]{2}{*}{ Total } \\
\hline & Básica & $\begin{array}{l}\text { Comple- } \\
\text { mentaria }\end{array}$ & & & \\
\hline $\begin{array}{lllll}\text { Teruel } & \ldots & \ldots & \ldots & \ldots \\
\text { Toledo } & \ldots & \ldots & \ldots & \ldots \\
\text { Valencia... } & \ldots & \ldots & \ldots \\
\text { Valladolid } & \ldots & \ldots & \ldots \\
\text { Vizcaya } & \ldots & \ldots & \ldots & \ldots \\
\text { Zamora... } & \ldots & \ldots & \ldots \\
\text { Zaragoza } & \ldots & \ldots & \ldots \\
\end{array}$ & $\begin{array}{l}292,2 \\
535,7 \\
574,0 \\
322,2 \\
129,8 \\
329,9 \\
560,8\end{array}$ & $\begin{array}{l}374,9 \\
412,9 \\
430,2 \\
332,5 \\
300,8 \\
337,8 \\
463,7\end{array}$ & $\begin{array}{r}1.333,6 \\
1.730,1 \\
339,3 \\
904,8 \\
260,1 \\
964,5 \\
1.595,6\end{array}$ & $\begin{array}{r}846,2 \\
859,9 \\
2.089,0 \\
1.324,7 \\
752,5 \\
871,8 \\
952,9\end{array}$ & $\begin{array}{l}2.846,9 \\
3.536,6 \\
3.432,5 \\
2.884,2 \\
1.443,2 \\
2.504,0 \\
3.573,0\end{array}$ \\
\hline
\end{tabular}

La red nacional básica tiene un trazado radical, con centro en Madrid, desde donde irradia hacia Galicia y Asturias, Santander y Vascongadas, Cataluña, Valencia, Andalucía y Extremadura. La longitud media provincial de esta red es de $324 \mathrm{kms}$. y las Provincias que superan esta cifra son 24 , las que se agrupan en esta forma:

- Madrid.

- León, Zamora, Lugo, Oviedo, Burgos y Valladolid, en las rutas del noroeste y norte.

- Zaragoza, Huesca, Lérida y Barcelona, en la ruta del nordeste.

- Cuenca, Albacete y Valencia, en la ruta del este.

- Toledo, Ciudad Real y todas las andaluzas, salvo Huelva, en la ruta del sur.

- Badajoz y Cáceres, en la ruta del oeste.

La red nacional complementaria trata de corregir los defectos del trazado radial de la red básica estableciendo corredores de comunicación entre las grandes rutas de la red radial básica. La longitud provincial media de esta red es de $379 \mathrm{kms}$., cifra que es superada por 27 Provincias, que se hallan ubicadas de esta manera:

- La Coruña, Lugo y Oviedo, al norte.

- León, Burgos, Segovia y Avila, en el valle del Duero.

- La Submeseta Sur, comprendidas todas sus Provincias, salvo Albacete.

- El valle del Ebro, con las tres Provincias aragonesas y Lérida.

- Valencia y Murcia.

- Sevilla, Córdoba, Málaga, Granada y Jaén.

- Baleares y Santa Cruz de Tenerife. 
La red regional trata de cubrir las necesidades de carreteras dentro de cada Región, enlazando, a su vez, a las Regiones colindantes. La longitud provincial media de esta red se cifra en $895 \mathrm{kms.}$ cifra superada por 27 Provincias que, geográficamente, se distribuyen así:

- Pontevedra, La Coruña, Lugo, Oviedo y Santander, al noroeste y norte.

- León, Zamora, Salamanca, Valladolid, Palencia y Burgos, en el valle del Duero.

- La Submeseta Sur, excepto Madrid.

- Aragón.

- Gerona, Alicante y Murcia, en el litoral mediterráneo.

- Jaén, Córdoba y Sevilla.

La red provincial, formada por las carreteras provinciales y los caminos vecinales, completa la red de carreteras. La longitud promedia provincial es de $1.335 \mathrm{kms}$., cifra superada sólo por 20 Provincias:

- Galicia y Asturias.

- León, Salamanca y Burgos.

- Navarra.

- Extremadura, Ciudad Real y Albacete.

- Barcelona, Valencia y Murcia.

- Jaén, Córdoba, Sevilla y Málaga.

En conjunto, la red de carreteras presenta una longitud media provincial de $2.932 \mathrm{kms}$., superada por 24 Provincias, que se distribuyen geográficamente en esta forma:

- Pontevedra, La Coruña, Lugo y Oviedo.

- León, Salamanca y Burgos.

- La Submeseta Sur, excepto Madrid.

- Aragón.

- Barcelona, Valencia y Murcia.

- Jaén, Granada, Córdoba y Sevilla.

Como se observa, existe una gran similitud en la configuración geográfica de las Provincias, que superan la superficie media provincial en cada una de las redes consideradas, lo que demuestra el carácter coordinado de las mismas, constituyendo un todo armónico al servicio del transporte. 
Esta red de carreteras sirve a un parque de vehiculos, en creciente progresión, que en los años 1973 y 1978 se distribuía, por clases de vehículos, en la siguiente forma:

\begin{tabular}{|c|c|c|}
\hline & 1973 & 1978 \\
\hline $\begin{array}{llllllllll}\text { Motocicletas } & \ldots & \ldots & \ldots & \ldots & \ldots & \ldots & \ldots & \ldots & \ldots\end{array}$ & 1.199 .854 & 1.170 .527 \\
\hline 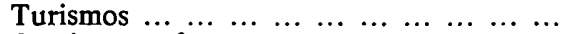 & 3.803 .659 & 6.530 .428 \\
\hline Camiones y furgonetas $\ldots \ldots \ldots \ldots \ldots$ & 887.981 & 1.189 .993 \\
\hline Autocares y microbuses $\ldots \ldots \ldots \ldots \ldots \ldots$ & 35.916 & 41.569 \\
\hline $\begin{array}{lllllllllll}\text { Tractore } & \ldots & \ldots & \ldots & \ldots & \ldots & \ldots & \ldots & \ldots & \ldots & \ldots\end{array}$ & 9.409 & 20.111 \\
\hline $\begin{array}{lllllllll}\text { Totales } & \ldots & \ldots & \ldots & \ldots & \ldots & \ldots & \ldots & \ldots\end{array}$ & 5.936 .819 & 8.952 .628 \\
\hline $\begin{array}{lllllllllll}\text { Remolques } & \ldots & \ldots & \ldots & \ldots & \ldots & \ldots & \ldots & \ldots & \ldots & \ldots\end{array}$ & 12.961 & 13.793 \\
\hline $\begin{array}{lllllllll}\text { Semirremolques } & \ldots & \ldots & \ldots & \ldots & \ldots & \ldots & \ldots & \ldots\end{array}$ & 12.135 & 27.695 \\
\hline
\end{tabular}

La cifra de vehículos automóviles se ha incrementado, en el período 1973-1978, en más de la mitad. Por clases, los turismos aumentaron en más del 70 por $100(71,7)$, los camiones y furgonetas en una tercera parte, los autocares y microbuses en una sexta parte y los tractores se duplicaron. Tan sólo disminuyó el número de motocicletas. En cuanto el material no automóvil, los remolques aumentaron tan sólo un 6 por 100, mientras que los semirremolques se duplicaron, a lo menos.

A ellos hay que añadir los casi 10 millones de coches de turismo y autocares entrados en España, en 1977, por los 23 millones de turistas extranjeros y españoles residentes en el extranjero, en el año citado.

Dentro del tráfico que genera el citado parque de vehículos nacional y extranjero, hay que destacar el que generan las 69.333 empresas dedicadas al transporte público de viajeros por carretera, con sus 100.522 vehículos, con 1.455 .175 plazas, en 1978 , que transportaron 576 millones (1977) de viajeros; los 13.496 vehículos de transporte discrecional de viajeros, de radio nacional, comarcal o local, con 622.597 plazas, en 1978, y las 144.802 empresas de servicio público de transporte de mercancias por carretera, con 255.109 vehículos, con una carga de 2,4 millones de Tm., más los 226.942 vehículos de transporte discrecional de mercancías, de radio nacional, comarcal y local, con 1,9 millones de Tm. de carga, también en 1978.

El Instituto Nacional de Estadística elabora anualmente la Estadística del transporte regular de viajeros por carretera, y en cooperación con el Ministerio de Obras Públicas y Urbanismo, realizó, en los años 1969 y 1975, sendas encuestas sobre el transporte 
público regular de mercancías por carretera. En el número 197 (enero-marzo 1978) de esta REvisTa DE Estudios DE LA Vma Local se comentaron los resultados de la encuesta de 1969.

Mediante sus encuestas de control de aforo en las carreteras españolas, realizadas por el Ministerio de Obras Públicas y Urbanismo, se conoce la intensidad media diaria que en ellas se registra. En 1978, los tramos de intensidad máxima, superior a 10.000 vehículos día, eran las siguientes:

- En el centro, Madrid, en salidas hacia el noroeste, norte, nordeste, sur y oeste.

- Al noroeste y norte, las salidas de El Ferrol y La Coruña, la carretera de Santiago a Vigo; el triángulo Oviedo-Avilés-Gijón; Torrelavega-Santander; salidas de Bilbao, San Sebastián, Vitoria, Burgos y Pamplona.

- Al nordeste, las salidas de Zaragoza y de Lérida a Barcelona, Tarragona-Barcelona y Barcelona-Gerona.

- Al este, la carretera litoral, con la autopista paralela, desde Tarragona a Murcia, comprendidas las salidas de Valencia, Alicante y Murcia.

- Al sur, las salidas de Almería, Granada y Málaga, con prolongación por toda la costa-Málaga-Algeciras-, y de Córdoba, Sevilla, Cádiz y, en menor longitud, de Huelva.

- En las islas Baleares, los tramos desde Palma de Mallorca a Manacor y Alcudia. Y en Canarias, desde Santa Cruz de Tenerife hacia el sur y en Las Palmas, desde la capital al aeropuerto.

\section{EL TRANSPORTE URBANO}

El vehículo privado acapara un alto porcentaje de los viajes motorizados en zona urbana. Este fenómeno, iniciado en la década de los sesenta, se ha intensificado en los últimos años, y ni siquiera la crisis de la energía de 1973 ha provocado cambios apreciables en dicha tendencia. El enorme desarrollo de la urbanización, registrado en España en los últimos decenios, y el reciente fenómeno de la suburbanización favorecen el uso frecuente del vehículo privado frente al transporte público colectivo.

Las características más importantes del transporte público urbano colectivo, referidas al conjunto de las ciudades españolas con más de 5.000 habitantes, se resumen en los siguientes datos: 


\begin{tabular}{|c|c|c|}
\hline & 1965 & 1977 \\
\hline Viajeros transportados (millones) $\ldots \ldots \ldots \ldots$ & 2.623 & 2.443 \\
\hline 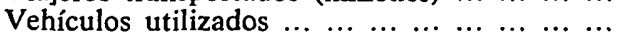 & 5.702 & 9.746 \\
\hline 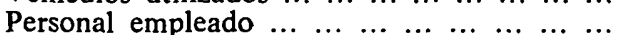 & 33.306 & 41.246 \\
\hline Gastos (millones de pesetas) $\ldots \ldots \ldots \ldots \ldots$ & 3.964 & 33.829 \\
\hline 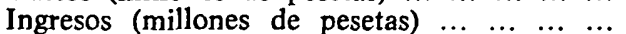 & 4.453 & 24.260 \\
\hline
\end{tabular}

Con un incremento de vehículos cifrado en el 71 por $100 \mathrm{y}$ un aumento del personal empleado del 24 por 100, se registra, en el período 1965-1977, una disminución del número de viajeros transportados del 7 por 100.

En dicho período, el parque de vehículos registró un paulatino incremento. La evolución del personal empleado fue más irregular, con un débil aumento hasta 1976, y un crecimiento más fuerte en 1977. La cifra de viajeros transportados crece hasta 1973 y comienza a disminuir a partir de 1974, decrecimiento que se acentúa en los años siguientes.

Los ingresos y gastos de las empresas de transporte urbano presentan una desigual evolución a lo largo del período. Los ingresos superan a los gastos hasta 1970. A partir de este año la situación resulta desfavorable y las explotaciones son deficitarias.

En orden a la importancia relativa de cada modo de transporte, nos limitaremos preferentemente al análisis de las redes de autobuses y de los metropolitanos de Madrid y Barcelona, puesto que en 1977 había desaparecido la totalidad de las redes de tranvías y sólo quedaban las líneas de trolebuses de Bilbao y La Coruña.

La evolución de las redes de transporte urbano, basada en las líneas de autobuses, puede deducirse de las siguientes cifras:

\begin{tabular}{|c|c|c|}
\hline & 1965 & 1977 \\
\hline Longitud de las líneas $(\mathrm{km}.) \ldots .$. & 3.968 & 7.857 \\
\hline Viajeros transportados (millones) & 1.252 & 1.682 \\
\hline Vehículos utilizados $\ldots \ldots \ldots \ldots \ldots$ & 3.746 & 8.566 \\
\hline Personal empleado $\ldots \ldots \ldots \ldots \ldots \ldots$ & 17.973 & 33.708 \\
\hline Gastos (millones de pesetas) ... & 2.176 & 28.088 \\
\hline Ingresos (millones de pesetas) $\ldots \ldots \ldots$ & 2.434 & 19.255 \\
\hline Viajeros por $\mathrm{km}$. de línea (millones) ... & 315 & 215 \\
\hline Viajeros por vehículo (millones) ... ... ... & 334 & 197 \\
\hline Ingresos por viajero (peestas) $\ldots \ldots \ldots \ldots$ & 1,94 & 11,41 \\
\hline Coeficiente de explotación $\ldots \ldots \ldots \ldots \ldots$ & 89,40 & 145,87 \\
\hline
\end{tabular}

Como se advierte, en el período $1965-1977$ se ha duplicado la longitud de las líneas y el personal empleado.

Los viajeros transportados aumentaron en una tercera parte hasta 1970, y la explotación resultó rentable hasta dicho año. 
En los años siguientes los gastos han superado a los ingresos de manera creciente y la explotación ha pasado a ser deficitaria. El número de viajeros por línea ha disminuido en una tercera parte y el de viajeros por vehículo en un 41 por 100; y aunque los ingresos por vehículo se han decuplicado, el coeficiente de explotación se ha incrementado en un 63 por 100 .

A continuación se presenta la tabla III, relativa al tráfico urbano por Provincias en 1977, por lo que a las líneas de autobuses se refiere.

TABLA III.-VIAIEROS TRANSPORTADOS Y PROMEDIO DIARIO DE VIAIEROS EN LAS LINEAS DE AUTOBUSES

\begin{tabular}{|c|c|c|}
\hline$P R O V I N C I A S$ & $\begin{array}{l}\text { Viajeros } \\
\text { (miles) }\end{array}$ & $\begin{array}{c}\text { Promedio } \\
\text { diario } \\
\text { de viajeros }\end{array}$ \\
\hline $\begin{array}{llllllll}\text { Totales } & \ldots & \ldots & \ldots & \ldots & \ldots & \ldots & \ldots\end{array}$ & 1.682 .301 & 4.609 .043 \\
\hline $\begin{array}{lllllllllll}\text { Alava } & \ldots & \ldots & \ldots & \ldots & \ldots & \ldots & \ldots & \ldots & \ldots & \ldots\end{array}$ & 9.961 & 27.290 \\
\hline Albacete $\ldots \ldots \ldots \ldots \ldots \ldots$ & 2.223 & 6.090 \\
\hline $\begin{array}{lllllllllllll}\text { Alicante } & \ldots & \ldots & \ldots & \ldots & \ldots & \ldots & \ldots & \ldots & \ldots & \ldots\end{array}$ & 36.236 & 99.270 \\
\hline $\begin{array}{ccccccccc}\text { Almería } & \ldots & \ldots & \ldots & \ldots & \ldots & \ldots & \ldots & \ldots\end{array} \ldots$ & 8.379 & 22.956 \\
\hline $\begin{array}{llllllllllll}\text { Avila } & \ldots & \ldots & \ldots & \ldots & \ldots & \ldots & \ldots & \ldots & \ldots & \ldots & \ldots\end{array}$ & 611 & 1.674 \\
\hline $\begin{array}{ccccccccc}\text { Badajoz } & \ldots & \ldots & \ldots & \ldots & \ldots & \ldots & \ldots & \ldots\end{array} \ldots$ & 4.396 & 12.044 \\
\hline $\begin{array}{cccccccccc}\text { Baleares } & \ldots & \ldots & \ldots & \ldots & \ldots & \ldots & \ldots & \ldots & \ldots\end{array}$ & 37.996 & 104.099 \\
\hline $\begin{array}{cccccccccc}\text { Barcelona } & \ldots & \ldots & \ldots & \ldots & \ldots & \ldots & \ldots & \ldots & \ldots\end{array}$ & 287.063 & 786.474 \\
\hline $\begin{array}{llllllllllll}\text { Burgos } & \ldots & \ldots & \ldots & \ldots & \ldots & \ldots & \ldots & \ldots & \ldots & \ldots\end{array}$ & 13.937 & 38.184 \\
\hline $\begin{array}{lllllllllll}\text { Cáceres } & \ldots & \ldots & \ldots & \ldots & \ldots & \ldots & \ldots & \ldots & \ldots & \ldots\end{array}$ & 3.754 & 10.285 \\
\hline 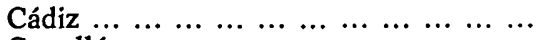 & 39.899 & 109.312 \\
\hline $\begin{array}{llllllllll}\text { Castellón } & \ldots & \ldots & \ldots & \ldots & \ldots & \ldots & \ldots & \ldots & \ldots\end{array}$ & 2.820 & $\mathbf{7 . 7 2 6}$ \\
\hline $\begin{array}{llllllllll}\text { Ciudad Real } & \ldots & \ldots & \ldots & \ldots & \ldots & \ldots & \ldots & \ldots\end{array}$ & 1.710 & 4.685 \\
\hline $\begin{array}{lllllllllll}\text { Córdoba } & \ldots & \ldots & \ldots & \ldots & \ldots & \ldots & \ldots & \ldots & \ldots\end{array}$ & 19.404 & 53.162 \\
\hline Coruña, La $\ldots \ldots \ldots \ldots \ldots$ & 23.831 & 65.290 \\
\hline $\begin{array}{llllllllllll}\text { Cuenca } & \ldots & \ldots & \ldots & \ldots & \ldots & \ldots & \ldots & \ldots & \ldots & \ldots\end{array}$ & 1.033 & 2.930 \\
\hline 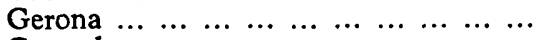 & 2.550 & 6.986 \\
\hline $\begin{array}{lllllllllll}\text { Granada } & \ldots & \ldots & \ldots & \ldots & \ldots & \ldots & \ldots & \ldots & \ldots\end{array}$ & 23.107 & 63.307 \\
\hline $\begin{array}{llllllllll}\text { Guadalajara } & \ldots & \ldots & \ldots & \ldots & \ldots & \ldots & \ldots & \ldots & \ldots\end{array}$ & 3.187 & 8.732 \\
\hline $\begin{array}{lllllllllll}\text { Guipúzcoa } & \ldots & \ldots & \ldots & \ldots & \ldots & \ldots & \ldots & \ldots & \ldots & \ldots\end{array}$ & 36.629 & 100.354 \\
\hline $\begin{array}{llllllllllll}\text { Huelva } & \ldots & \ldots & \ldots & \ldots & \ldots & \ldots & \ldots & \ldots & \ldots & \ldots\end{array}$ & 14.561 & 40.140 \\
\hline $\begin{array}{llllllllllll}\text { Huesca } & \ldots & \ldots & \ldots & \ldots & \ldots & \ldots & \ldots & \ldots & \ldots & \ldots\end{array}$ & (1) & (1) \\
\hline $\begin{array}{llllllllllll} & \text { Jaén } & \ldots & \ldots & \ldots & \ldots & \ldots & \ldots & \ldots & \ldots & \ldots & \ldots\end{array}$ & 4.093 & 11.214 \\
\hline 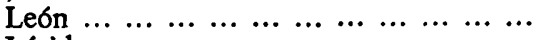 & 3.515 & 9.630 \\
\hline $\begin{array}{lllllllllll}\text { Lérida } & \ldots & \ldots & \ldots & \ldots & \ldots & \ldots & \ldots & \ldots & \ldots & \ldots\end{array}$ & 6.213 & 17.022 \\
\hline $\begin{array}{llllllllll}\text { Logroño } & \ldots & \ldots & \ldots & \ldots & \ldots & \ldots & \ldots & \ldots & \ldots\end{array}$ & 3.012 & 8.252 \\
\hline $\begin{array}{llllllllll}\text { Lugo } & \ldots & \ldots & \ldots & \ldots & \ldots & \ldots & \ldots & \ldots & \ldots\end{array}$ & 1.833 & 5.022 \\
\hline $\begin{array}{lllllllllll}\text { Madrid } & \ldots & \ldots & \ldots & \ldots & \ldots & \ldots & \ldots & \ldots & \ldots & \ldots\end{array}$ & 489.625 & 1.341 .438 \\
\hline $\begin{array}{lllllllllll}\text { Málaga } & \ldots & \ldots & \ldots & \ldots & \ldots & \ldots & \ldots & \ldots & \ldots & \ldots\end{array}$ & 59.099 & 161.915 \\
\hline $\begin{array}{llllllllllll}\text { Murcia } & \ldots & \ldots & \ldots & \ldots & \ldots & \ldots & \ldots & \ldots & \ldots & \ldots\end{array}$ & 11.736 & 32.153 \\
\hline $\begin{array}{llllllllll}\operatorname{Navarra} & \ldots & \ldots & \ldots & \ldots & \ldots & \ldots & \ldots & \ldots & \ldots\end{array}$ & 28.874 & 79.107 \\
\hline $\begin{array}{lllllllllll}\text { Orense } & \ldots & \ldots & \ldots & \ldots & \ldots & \ldots & \ldots & \ldots & \ldots & \ldots\end{array}$ & 4.382 & 12.005 \\
\hline $\begin{array}{llllllllllll}\text { Oviedo } & \ldots & \ldots & \ldots & \ldots & \ldots & \ldots & \ldots & \ldots & \ldots & \ldots\end{array}$ & 54.833 & 150.227 \\
\hline $\begin{array}{lllllllllll}\text { Palencia } & \ldots & \ldots & \ldots & \ldots & \ldots & \ldots & \ldots & \ldots & \ldots & \ldots\end{array}$ & $\begin{array}{r}2.042 \\
50550\end{array}$ & $\begin{array}{r}5.594 \\
170.097\end{array}$ \\
\hline $\begin{array}{lllllllll} & \text { Palmas, Las } & \ldots & \ldots & \ldots & \ldots & \ldots & \ldots & \ldots\end{array}$ & 50.550 & 138.493 \\
\hline
\end{tabular}




\begin{tabular}{|c|c|c|}
\hline$P R O V I N C I A S$ & $\begin{array}{c}\text { Viajeros } \\
\text { (miles) }\end{array}$ & $\begin{array}{c}\text { Promedio } \\
\text { diario } \\
\text { de viajeros }\end{array}$ \\
\hline 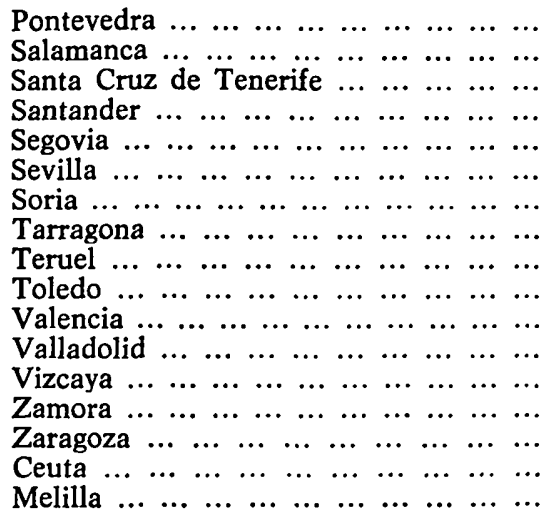 & $\begin{array}{r}24.761 \\
5.280 \\
10.635 \\
24.353 \\
3.099 \\
77.393 \\
375 \\
5.674 \\
891 \\
4.094 \\
83.072 \\
20.577 \\
46.624 \\
2.114 \\
68.072 \\
4.817 \\
7.286\end{array}$ & $\begin{array}{r}67.838 \\
14.466 \\
29.137 \\
66.721 \\
8.490 \\
212.036 \\
1.027 \\
15.545 \\
2.441 \\
11.216 \\
227.595 \\
56.375 \\
127.737 \\
5.792 \\
186.499 \\
13.197 \\
19.962\end{array}$ \\
\hline
\end{tabular}

Del examen de las cifras anteriores se deduce que existe una marcada diferencia de unas a otras Provincias, en orden a la importancia del transporte urbano, debido al desigual peso demográfico y económico en el conjunto de la Nación. Si tomamos como punto de referencia el promedio provincial de viajeros transportados en conjunto y diariamente- 32.351 y 88.635 , respectivamente-, observamos que tan sólo 13 Provincias superan estos promedios. Destacan, en primer término, Madrid y Barcelona, con el 29 y el 17 por 100 , respectivamente, de viajeros transportados y de viajeros promedio diario, sobre el total del tráfico urbano de autobuses. Las 11 Provincias restantes se distribuyen geográficamente así:

- Norte: Oviedo, Vizcaya, Guipúzcoa, Zaragoza.

- Este: Valencia, Alicante, Baleares.

- Sur: Sevilla, Málaga, Cádiz, Las Palmas.

En los mismos años, 1965 y 1977, los metropolitanos presentaban las siguientes características estructurales y de actividad: 


\begin{tabular}{|c|c|c|}
\hline & 1965 & 1977 \\
\hline Longitud de las líneas $(\mathrm{km}.) \ldots \ldots$ & 67 & 112 \\
\hline Viajeros transportados (millones) $\ldots \ldots \ldots$ & 729 & 761 \\
\hline Vehículos utilizados $\ldots \ldots \ldots \ldots \ldots$ & 554 & 1.150 \\
\hline $\begin{array}{llllllll}\text { Personal empleado } & \ldots & \ldots & \ldots & \ldots & \ldots & \ldots & \ldots\end{array}$ & 5.621 & 7.473 \\
\hline $\begin{array}{lllllllll}\text { Gastos (millones) } & \ldots & \ldots & \ldots & \ldots & \ldots & \ldots & \ldots & \ldots\end{array}$ & 702 & 5.695 \\
\hline 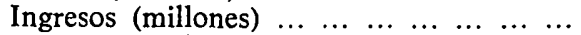 & 979 & 4.974 \\
\hline Viajeros por $\mathrm{km}$. de línea (millones) $\ldots \ldots$ & 10.880 & 6.827 \\
\hline Viajeros por vehículo (millones) $\ldots \ldots \ldots$ & 1.316 & 653 \\
\hline Ingresos por viajero $\left[\begin{array}{llllllll} & \ldots & \ldots & \ldots & \ldots & \ldots & \ldots & \ldots\end{array}\right.$ & 1,34 & 6,62 \\
\hline 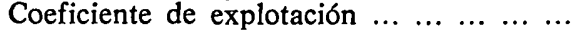 & 71,70 & 114,50 \\
\hline
\end{tabular}

Observamos que la longitud de las líneas de los metropolitanos se ha incrementado, en el período 1965-1977, en las dos terceras partes, el número de vehículos ha aumentado en más del 100 por 100 y que el personal empleado se incrementó, asimismo, en una tercera parte; sin embargo, el número de viajeros transportados ha aumentado tan sólo en un 4 por 100. La explotación ha sido rentable hasta 1975 y a partir de 1976 los gastos han superado a los ingresos, los viajeros por $\mathrm{km}$. han descendido en una tercera parte y los viajeros por vehículo en la mitad, y aunque los ingresos por viajero se han multiplicado por cinco, el coeficiente de explotación ha aumentado en un 60 por 100 .

Por lo que se refiere a Madrid y Barcelona, las cifras correspondientes al tráfico urbano de autobuses y metropolitano, han sido, en los años que se citan:

AUTOBuses

\section{Madrid}

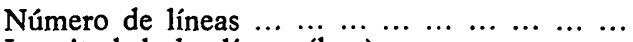

Longitud de las líneas $(\mathrm{km}.) \ldots \ldots \ldots \ldots \ldots$

Viajeros transportados (millones) $\ldots \ldots \ldots \ldots$

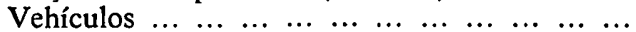

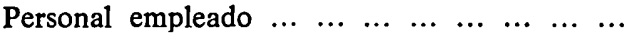

Barcelona

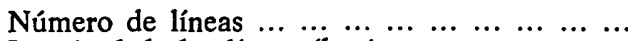

Longitud de las líneas $(\mathrm{km}.) \ldots \ldots \ldots \ldots$

Viajeros transportados (millones) $\ldots \ldots \ldots \ldots$

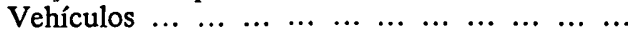

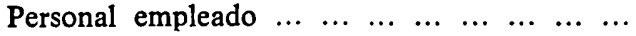

Metropolitanos

\section{Madrid}

Número de líneas $\ldots \ldots \ldots \ldots \ldots \ldots \ldots$

Longitud de las líneas $(\mathrm{km}.) \quad \ldots \ldots \ldots \ldots$

Estaciones $\quad \ldots \quad \ldots \quad \ldots \ldots \ldots \ldots$

Viajeros transportados (millones) $\quad \ldots \ldots \ldots \ldots$

Personal empleado
1971

$\begin{array}{rrr}1971 & & 1978 \\ & & \\ 71 & & 127 \\ 459 & & 1.046 \\ 336 & & 428 \\ 1.228 & & 1.701 \\ 7.086 & & 7.266 \\ & & \\ 53 & & 115 \\ 350 & & 662 \\ 294 & & 272 \\ 806 & & 1.128 \\ 6.335 & & 6.430\end{array}$




\begin{tabular}{|c|c|c|}
\hline & 1971 & 1978 \\
\hline \multicolumn{3}{|l|}{ Barcelona } \\
\hline 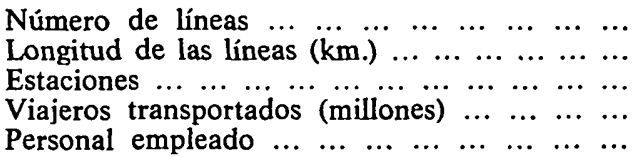 & $\begin{array}{r}3 \\
30 \\
45 \\
234 \\
2.025\end{array}$ & $\begin{array}{r}5 \\
48 \\
72 \\
257 \\
2.992\end{array}$ \\
\hline
\end{tabular}

En el período 1971-1978, en cuanto a los autobuses se refiere, en Madrid, aunque el número de líneas se incrementó en las cuatro quintas partes, su longitud más que se duplicó, el número de vehículos empleados aumentó en una tercera parte y el personal empleado se incrementó en el 2,5 por 100 , los viajeros transportados aumentaron en un 27 por 100.

En Barcelona, en el mismo período, el número de líneas de autobuses más que se duplicó, su longitud aumentó en un 90 por 100 , el número de vehículos en el $40 \mathrm{y}$ el personal empleado en tan sólo el 1,5 por 100; sin embargo, se registró una disminución del 7,5 por 100 en el número de viajeros.

Respecto a los metropolitanos, en Madrid aumentó la longitud de sus líneas en el 22 por 100, el número de estaciones y el personal empleado en una sexta parte, si bien el número de viajeros disminuyó en un 17 por 100.

En Barcelona, la longitud de sus líneas y el número de estaciones aumentaron en un 60 por 100 y el personal empleado en un 48, si bien el número de viajeros aumentó tan sólo en un 10 por 100 .

En conjunto, el personal empleado en los autobuses y el metropolitano de Madrid fue de 11.169 en 1971 y de 11.948 en 1978, con un aumento tan sólo del 7 por 100, mientras que en Barcelona era, en 1971, de 8.360, y en 1978, de 9.422, con un aumento del 13 por 100 .

Los viajeros transportados por ambos modos de transporte fueron, en Madrid, de 877 millones en 1971 y de 875 en 1978, y en Barcelona, de 528 millones en 1971 y de 529 en 1978, cantidades prácticamente iguales en ambos momentos.

La participación del metropolitano en el transporte colectivo de viajeros de Madrid, fue en 1973 del 57,23 por 100, disminuyendo al 51,09 en 1978, mientras que en Barcelona fue del 44,42 en 1973, subiendo al 48,58 en 1978 .

Los viajeros de transporte urbano, según modos de transporte, en las ciudades de Bilbao, Sevilla, Valencia y Zaragoza, poblaciones mayores de 400.000 habitantes, fue, en los años de referencia y expresado en porcentajes, como sigue: 


\begin{tabular}{|c|c|c|}
\hline & 1970 & 1977 \\
\hline \multicolumn{3}{|l|}{ Bilbao } \\
\hline $\begin{array}{lllllllllll}\text { Autobuses } & \ldots & \ldots & \ldots & \ldots & \ldots & \ldots & \ldots & \ldots & \ldots & \ldots \\
\text { Trolebuses } & \ldots & \ldots & \ldots & \ldots & \ldots & \ldots & \ldots & \ldots & \ldots & \ldots\end{array}$ & $\begin{array}{l}75 \\
25\end{array}$ & $\begin{array}{r}99 \\
1\end{array}$ \\
\hline \multicolumn{3}{|l|}{ Sevilla } \\
\hline $\begin{array}{llllllllllll}\text { Autobuses } & \ldots & \ldots & \ldots & \ldots & \ldots & \ldots & \ldots & \ldots & \ldots & \ldots\end{array}$ & 100 & 180 \\
\hline \multicolumn{3}{|l|}{ Valencia } \\
\hline 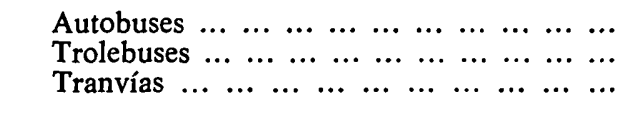 & $\begin{array}{r}73 \\
19 \\
8\end{array}$ & $\underline{100}$ \\
\hline \multicolumn{3}{|l|}{ Zaragoza } \\
\hline 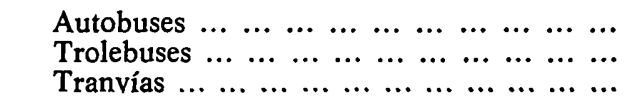 & $\begin{array}{l}45 \\
10 \\
45\end{array}$ & $\underline{100}$ \\
\hline
\end{tabular}

En el período 1970-1977 se observa una tendencia hacia la utilización de autobuses, con carácter general, prescindiendo de los tranvías y trolebuses, $y$ un descenso generalizado también de viajeros que han utilizado los transportes públicos, que se cifra, en Bilbao, en el 32 por 100, en Sevilla, en el 35, el 28 en Valencia y el 54 por 100 en Zaragoza. 
REVISTA

DE

ESTUDIOS

DE LA

VIDA LOCAL

\section{JURISPRUDENCIA}


REVL-1980, núm. 206. BALLESTER ROS, IGNACIO. IMPORTANCIA RELATIVA DE LOS DISTINT... REVL-1980, núm. 206. BALLESTER ROS, IGNACIO. IMPORTANCIA RELATIVA DE LOS DISTINT... 